\title{
De la pulsión a la relación: El nacimiento del psicoterapeuta ${ }^{1}$
}

\author{
Hélder Chambel ${ }^{2}$ \\ PsiRelacional, Lisboa, Portugal
}

Ser psicoterapeuta es un desafío permanente. La psicoterapia es una experiencia relacional y emocional intensa. No tenemos conciencia de todo lo que sucede en la relación terapéutica. El estudio de la teoría psicoanalítica, nuestra experiencia de vida, nuestra análisis y supervisión nos dan un marco para intentar comprender la experiencia relacional terapéutica. En este encuentro de matrices relacionales el psicoterapeuta define una actitud relacional derivada de su subjetividad. Una de las dimensiones de esa subjetividad, quizás la más fácil de pensar, es su inspiración teórica. Se identifican actitudes relacionales del psicoterapeuta derivadas de la teoría psicoanalítica y se evidencian sus implicaciones clínicas.

Palabras clave: psicoterapia; relación terapéutica; psicoanálisis relacional.

Being a psychotherapist is a permanent challenge. Psychotherapy is an intense relational and emotinal experience. We are not aware of everything that happens in the therapeutic relationship. The study of psychoanalytic theory, our experience of life, our analysis and supervisions give us a framework to try to understand the therapeutic relational experience. In this encounter of relational matrices, the psychotherapist defines a relational attitude derived from his subjectivity. One of the dimensions of this subjectivity, perhaps the easiest to think, is his theoretical inspiration. We identify the relational attitudes of the psychotherapist derived from the psychoanalytic theory and evidence of its clinical implications.

Key Words: psychotherapy; therapeutic relationship; relational psychoanalysis.

English Title: From the drive to the relationship. The birth of the psychotherapist.

\section{Cita bibliográfica / Reference citation:}

Chambel, H. (2019). De la pulsión a la relación. El nacimiento del psicoterapeuta. Clínica e Investigación Relacional, 13 (1): 109-133. [ISSN 1988-2939] [Recuperado de www.ceir.info ] DOI: 10.21110/19882939.2019.130108

\footnotetext{
${ }^{1}$ Versión revisada del trabajo presentado en las /l Jornadas de Psicanálise Relacional. Fac. Psicologia, Lisboa, 1719 de Mayo de 2018. Versión castellana preparada por el autor, con revisiones editoriales menores a cargo de la redacción de CeIR.

2 Psicólogo Clínico, Miembro Efectivo de la Asociación de Psicoanálisis Relacional - PsiRelacional. helderchambel@gmail.com
} 
"Yo soy porque tú eres"

"Sólo puedo ser a través de las otras personas"

De la filosofía Africana Ubuntu

Ser psicoterapeuta es un desafío permanente, como dice Thomas Ogden (2010), el psicoanálisis es una experiencia emocional vivida, como tal no puede ser registrada, explicada o contada, muchas veces no puede ser comprendida. El encuentro terapéutico convoca dimensiones humanas pre-verbales, donde las palabras son demasiado cortas para describir la multiplicidad de acontecimientos que se entrelazan entre psicoterapeuta y paciente. No es posible, más allá de lo obvio, tener una verdad absoluta sobre la dinámica relacional, sobre nuestra subjetividad ni sobre la subjetividad del paciente. Navegamos en un territorio complejo donde no tenemos conciencia de mucho de lo que sucede.

Sin embargo, no se trata de navegar a la deriva, tenemos nuestras brújulas, un conjunto de principios organizadores que permiten pensar la relación terapéutica. Tenemos nuestra experiencia de vida en un determinado tiempo social y cultural, un tiempo constituyente de nuestra subjetividad. En mi subjetividad, encuentro una cierta manera de ser y estar constituida con mis relaciones significativas, encuentro mi análisis, las supervisiones que hice, el estudio de la teoría psicoanalítica. Es de este universo, de la ecuación infinita entre todas las dimensiones conscientes e inconscientes de mi subjetividad, que resulta la respuesta que pienso ser la mejor para ayudar al paciente, y para cada paciente la respuesta es diferente, ya que también resulta de la articulación de mi subjetividad con otra subjetividad.

Si desea analizar aisladamente las dimensiones constituyentes de mis actitudes, y no creo que eso sea posible, por ejemplo, la lectura que hago de las teorías psicoanalíticas está influida por otras dimensiones de mi subjetividad, pero si lo quiero hacer como forma de pensar, lo más simple de aislar es la teoría psicoanalítica que me sirve de referencia. Es lo que voy a intentar hacer, identificar en la teoría psicoanalítica fundamentos para las actitudes relacionales del psicoterapeuta.

Este análisis parte de tres autores: Freud, Ferenczi y Winnicott, articulados con autores del psicoanálisis relacional. Destaco tres orientaciones teóricas que fundamentan las actitudes relacionales del psicoterapeuta, una dimensión de psicoterapeuta objeto de la pulsión del paciente, idea Freudiana, otra dimensión de psicoterapeuta que repara una falla ambiental, profundizada por Winnicott, y una idea de psicoterapeuta como otro relacional provisto de 
subjetividad y relacionalidad trabajada por autores del psicoanálisis intersubjetivo y relacional. Sé que es reduccionista, que deja de lado prespectivas diferentes e intermedias, pero puede ser organizador, por lo menos para mí.

Veamos la idea de una dimensión de terapeuta objeto. En el tiempo inicial del psicoanálisis (Freud, 1893, 1894, 1895a, 1896a) la idea es que hay un incidente que provoca los síntomas histéricos, un abuso sexual del adulto al niño, este traumatismo actúa como un cuerpo extraño al paciente en la medida en que no tiene recuerdo de él, una parte de lo consciente, constituido por afectos penosos, es disociado. El recuerdo del acontecimiento genera angustia y por eso es reprimida, en la lectura de Freud el recuerdo tiene un quantum afectivo que necesita ser descargado. Supone la existencia de un sistema del tipo hidráulico en que las energías psíquicas buscan una homeostasis, la emoción asociada a la representación del trauma es más que el sistema tolera y se da, en el caso de la histeria, una conversión somática, la energía psíquica es transpuesta al cuerpo, es el retorno de lo reprimido en forma de síntoma.

El método clínico inicial es tomar conciencia del trauma, primero con el método catárquico bajo hipnosis, en la idea de descargar la energía del trauma a través de la verbalización, después con la asociación libre de ideas conjugada con la interpretación, así cree Freud que es posible deducir del discurso de las pacientes el trauma original y que es posible hacerlo consciente a través de la interpretación (Freud, 1904).

En un segundo momento teórico Freud deja de creer en la teoría de la seducción (carta a Fliess, 21/09/1897). Concluye que lo que las histéricas contaban no eran hechos, eran fantasías, nace la idea de realidad psíquica, el psicoanálisis, en un movimiento desde no fue un acontecimiento fue la fantasía de ese acontecimiento deja el factor externo como origen de la psicopatología para segundo plano y se centra en lo intrapsíquico y en el deseo inconsciente.

En La Interpretación de los Sueños (1900) Freud presenta un aparato psíquico con diferentes instancias en conflicto, el deseo inconsciente quiere irrumpir hacia la conciencia, la censura no autoriza porque el deseo entra en conflicto con las ideas morales y genera angustia, se da una negociación, el deseo sólo puede acceder a la conciencia disfrazado de forma que no cause angustia. La conciencia se transforma en un baile de máscaras en el que, por procesos como el desplazamiento, la condensación o el simbolismo, lo que es no es, la verdad no está en la palabra del paciente, está en su inconsciente. El hombre no es señor 
de sí, dijo Freud. Los sueños, los actos fallidos y los síntomas se explican mediante esta dinámica.

La teoría de la sexualidad (1905) concreta el contenido del inconsciente, la fantasía inconsciente está relacionada con el desarrollo psicosexual de donde sobresalía la idea del complejo de Edipo y de pulsión, fuente de la libido que dinamiza la inversión en el objeto.

Después de fundamentada y estabilizada la teoría en el conflicto intrapsíquico, y tras la presentación de casos clínicos ilustrativos (Dora, 1905; Hans; Hombre de las ratas, 1909; Leonardo da Vinci, 1910; Schreber, 1911), Freud escribe varios textos sobre recomendaciones técnicas (Freud 1912, 1912, 1913, 1914, 1915) que se convirtieron en la base de las reglas técnicas posteriormente desarrolladas y minuciosamente presentadas en manuales técnicos, por ejemplo Sandler (1973) o Etchegoyen (1986), aspiración a la que Freud, después de demostrar esa voluntad, renunció (Quinodoz, 2007).

El segundo movimiento teórico de Freud, centrado en la dinámica intrapsíquica, tiene consecuencias técnicas. La idea deja de ser concientizar el trauma originario y pasa a ser concientizar el deseo inconsciente de forma que se establecezcan nuevos equilibrios en la estructura de la mente del paciente. La teoría sexual fundamenta la interpretación que hace consciente el inconsciente y permite la curación.

Cuando el paciente no acepta la interpretación Freud cree que se trata de resistencia del paciente, se vea el texto La Negativa (1925), en que afirma que es exactamente la negación de una interpretación lo que la confirma, en esta lectura el psicoanálisis pasa a ser la aplicación técnica de una teoría al paciente.

En este contexto Freud se centra en la idea de transferencia y considera que los pacientes actualizan en la relación con el analista la inversión libidinal y agresiva originariamente dirigida a los padres en la infancia, lo que se convierte en un problema. La cura psicoanalítica es hecha por la conciencia de lo inconsciente, la deriva libidinal para una inversión en el analista impide la asociación libre y la interpretación. La transferencia es sin embargo pronto vista como una ventaja terapéutica en la medida en que su interpretación la concientiza y disuelve (Freud, 1912a).

En este marco Freud concluye sobre la posición relacional del analista. El analista se debe regir por la regla de la abstinencia, no gratificar los impulsos libidinales del paciente para no acentuar la transferencia, la relación con el paciente tendría que restringirse al período de análisis. La regla de la neutralidad exige que el analista sea neutro en relación a opiniones o juicios, remite al anonimato, el paciente no debe saber nada del analista. Todo 
esto con el objetivo de no interferir en las asociaciones libres e interpretaciones y de alejarse de la idea de sugerencia del analista.

En relación a la contratransferencia, el analista no debe sentir nada por el paciente, compara el análisis a una cirugía donde los afectos quedan fuera de la mente del médico. La técnica deriva de la teoría pulsional, del complejo de Edipo y del análisis de la transferencia. Es aquí donde el psicoterapeuta objeto se muestra, un cirujano desprovisto de afecto que hace consciente el inconsciente del paciente.

En el paso de un inconsciente de traumas reprimidos a un inconsciente de deseo Edípico, el papel del otro relacional cambia radicalmente, de agente psicopatologizante pasa a ser objetivo de la pulsión quedando desprovisto de subjetividad y relacionalidad. Como dice Mitchell (1998) aunque la articulación con lo externo no sea negada, por ejemplo, a través de identificaciones, introyecciones y proyecciones, la centralidad de la teoría pulsional está en el conflicto intrapsíquico.

Freud recomienda la retirada afectiva del espacio analítico. Queda por saber si es posible. Tal vez Freud, aunque constituyendo una subjetividad para el sujeto de la que deriva el síntoma, algo revolucionaria en esos momentos, queda rehén de una biología determinista y de la ciencia positivista, respetuoso lugar donde él imaginaba al psicoanálisis.

Muchos autores contemporáneos (Aron, 1996, Mitchell, 1997, Stolorow y Atwood, 2013) consideran la retirada relacional y emocional del analista del espacio terapéutico una imposibilidad comunicativa, un mito psicoanalítico. Consideran la metapsicología Freudiana reduccionista y mecanicista, un racionalismo cartesiano en una dinámica alimentada por la pulsión que desvaloriza el contexto y el otro relacional en la constitución y transformación de la persona. Freud se fundamenta en el mito de la mente aislada, en la psicología de una persona sola. Es la aceptación de la posibilidad de la mente aislada que permite a Freud fundamentar las reglas técnicas y la retirada afectiva del analista de la relación terapéutica (Atwood y Stolorow, 1992; Stolorow, Orange y Atwood, 2012).

Vale la pena recordar el cambio de paradigma científico que ocurre en las ciencias en general. En el tiempo de Freud el positivismo era la respuesta racional y objetiva a las explicaciones basadas en lo divino, sin embargo, más la ciencia se volvió más compleja. Hoy la verdad es relativa y contextual, hablamos de sistemas abiertos, complejos y no lineales donde el observador forma parte de la observación. La biología, la neurología y la genética dan mucha más importancia a la dialéctica con el contexto y las emociones en la constitución de la mente. La teoría de la ciencia posmoderna parece acompañar el cambio de paradigma que los autores relacionales introducen en el psicoanálisis (Coderch 2010, 2012, 2014, Martínez Ibáñez, 2017). La mente que era vista como derivada de un conjunto 
de estructuras predeterminadas en el individuo es ahora vista como emergente de la interacción de la biología con el medio, en ese sentido es un producto social (Mitchell, 1998).

Es interesante observar que muchas de nuestras actitudes y comportamientos en psicoterapia, hoy, resultan de las reglas técnicas definidas por Freud, fundamentadas por la teoría pulsional y por la posibilidad de asumir un terapeuta "pantalla en blanco".

En el tiempo inicial del psicoanálisis Ferenczi fue el único de los discípulos de Freud que, manteniéndose en el movimiento psicoanalítico, cuestionó la técnica psicoanalítica. A partir de 1921 inició una serie de experiencias terapéuticas, escribió un conjunto de textos y un diario clínico guardado por Balint y publicado sólo en 1985. En estos textos Ferenczi critica la técnica psicoanalítica llamando a primer plano terapéutico, el ambiente, el trauma, la relación y la afectividad. El diario clínico y la historia de su publicación tardía dice mucho sobre la relación entre Freud y Ferenczi al final de la vida de Ferenczi. Aron y Harris describen esta conmovedora historia en The Legacy of Sandor Ferenczi (1993). Aron (1996) considera que la publicación del diario sirvió de anclaje histórico para los autores que trabajaban en los conceptos relacionales y demuestra que la discusión teórico-clínica mantenida entre Freud y Ferenczi se transformaría en el telón de fondo de las controversias psicoanalíticas del futuro.

En El Niño y su Pulsión de Muerte (1929) Ferenczi dice que cuando el niño nace en un ambiente donde no es acogido con amor y protección percibe, consciente o inconscientemente, el rechazo y se vuelve desconfiado y pesimista, pierde la voluntad de vivir, puede incluso enfermarse y morir. Nos dice Ferenczi que son siempre conflictos con el mundo exterior que traumatizan y crean direcciones psicopatológicas en el desarrollo. En esta idea vuelve a la primera teoría de Freud, el medio pasa a agente en la constitución psíquica. En términos terapéuticos dice que lo que los neuróticos necesitan es que los dejemos, por primera vez, disfrutar de una infancia normal. Ferenczi pretende constituirse como la figura parental instigadora de la salud mental que el paciente no tuvo. La técnica se adapta a las necesidades del paciente, es elástica, y las necesidades son de afecto y comprensión. Ferenczi pretende sentir con y en esta sintonía percibir cuándo y de qué forma debe decir algo, llamó este movimiento de tacto psicológico.

En Confusión de lengua entre niños y adultos (1933) hace una crítica a lo que llama hipocresía psicoanalítica y defiende la sinceridad, la honestidad y la afectividad en el proceso terapéutico. Considera el trauma como fundamental en la constitución psíquica. Se analiza el trauma desestructurante a partir de la confusión de lenguajes entre la ternura del niño y la sexualidad del adulto y dice que la técnica analítica repite el mecanismo del trauma. 
Actúa como el desmentido traumático en la medida en que la vivencia traumática verbalizada por el paciente es interpretada como fantasía pulsional. El analista funciona como el adulto que desmiente el sentir del niño al mismo tiempo que la culpa, y desculpabiliza al agresor, obligando al niño a enterrar viva una parte de sí. Dice además que la postura del analista, fría, distante, indiferente y desconfiada de la inversión libidinal o agresiva del paciente, derivada de las reglas de abstinencia, anonimato y neutralidad, crean un ambiente en todo semejante a los ambientes traumáticos.

En este ambiente el analista se coloca en una relación de maestro / discípulo que infantiliza al paciente. Dice Ferenczi que el analista camina por encima del paciente como una divinidad no percibiendo que gran parte de la transferencia erótica o agresiva es provocada precisamente por esta postura del analista. Para agradar al analista, el paciente, tendrá que negar su sentir y enterrar viva una parte de sí pasando a funcionar en función del deseo del analista. Dice en la nota del 12 de junio del Diario Clínico: "Los pacientes prueban hasta donde el analista es capaz de aceptarlos como son o hasta qué punto se tiene que moldear al deseo del analista para ser aceptados". También considera que los pacientes perciben el inconsciente de los analistas y actúan en función de ello.

Ferenczi propone una técnica regresiva y maternal, donde predomina la interacción, lo infra-verbal, el sentir con, ambiente que debe permitir al analista entrar en contacto con el niño traumatizado que hay en el paciente. En su entender los resultados del psicoanálisis no se dan por la conciencia de la verdad inconsciente sino por la re-vivencia emocional de situaciones traumáticas en el aquí y ahora terapéutico. Las notas de 4 y 17 de agosto del diario clínico son interesantes, Ferenczi dice que no son sólo los pacientes los que proyectan su mundo interno sobre la relación analítica, también los psicoanalistas proyectan su mundo interno sobre los pacientes. Esta es la idea de Aron (1996) que en una perspectiva relacional dice que la interpretación del analista dice más sobre el mundo interno del analista que del paciente.

Freud discordaba de Ferenczi, le dice en la carta de 02/10/1932 "No quería perder la esperanza de que vendría a reconocer la inexactitud técnica de sus conclusiones, ya no creo que se corrija como yo me corregí hace una generación atrás". Ferenczi murió poco después. Cuando Freud escribió que sería imposible que el psicoanálisis lo olvidara, parecía adivinar lo que se preparaba para suceder, durante más de 40 años el psicoanálisis olvidó a Ferenczi. Pero incluso enterrado vivo Ferenczi no murió, varios autores inspirados en Ferenczi fueron haciendo su camino. La crítica de Ferenczi era devastadora, pero lo cierto es que lo externo y la relación primaria harían historia en las décadas siguientes y Ferenczi será considerado un precursor de los avances en psicoanálisis. 
Greenberg y Michell (1983) y Ávila Espada (2013) explican la reactualización de las ideas de Ferenczi por dos vías, por la Sociedad Británica (escuela de las relaciones de objeto y grupo de los independientes) y por la escuela de las Relaciones Interpersonales norteamericanas.

En cuanto a las teorías interpersonales norteamericanas, Ferenczi es una piedra angular en el origen de la tradición interpersonal, principalmente a través de Clara Thompson, que conjuntamente con Erich Fromm y Harry Sullivan, fundaron en los años 40 el William Alanson White Institute donde el valor de las relaciones interpersonales era central y los textos de Ferenczi objeto de culto. La escuela de las relaciones interpersonales daba importancia a los aspectos sociales y culturales en la formación del sujeto. Es de este contexto que va a surgir la idea del psicoanálisis relacional, Mitchel y Greenberg se formaron precisamente en el Willian Alanson White Institute y fue allí que pensaron escribir el libro Las Relaciones de Objeto en la Teoría Psicoanalítica (1983). Para muchos fue la pieza clave del cambio paradigmático que se opera en el psicoanálisis durante los años 80.

El libro es un estudio comparativo de escuelas psicoanalíticas que define una línea divisoria entre un modelo relacional y el modelo pulsional. La perspectiva relacional, en este primer momento, es el conjunto de teorías que no negando lo intrapsíquico dicen que es construido en una dialéctica con los otros, por oposición paradigmática al modelo pulsional en que el otro es principalmente objeto de la pulsión.

En relación con el grupo de los independientes de la Sociedad Británica, Balint, muy cercano a Ferenczi, fue a vivir a Londres en 1939 y estableció un puente entre Ferenczi y el grupo de los independientes, recuerde que este grupo se sitúa fuera de la guerra entre Klenianos y Freudianos, defendiendo una idea antidogmática y creativa de la formación, de la teoría y de la práctica psicoanalítica. Winnicott es de este grupo.

Freud moriría en 1939 y la técnica psicoanalítica no fue cuestionada hasta los años 50, cuando autores como Paula Heimann (1950) trabajan el concepto de contratransferencia como forma de comprender el mundo interno del analizando. La contratransferencia pasa a estar al servicio del análisis para la comprensión de lo intrapsíquico del paciente. Más detalladamente Winnicott (1947), Racker (1948) y Margaret Little (1951) consideran el papel de la subjetividad en el analista para la comprensión del acontecer terapéutico.

Estos avances están encuadrados en los trabajos Melanie Klein que a partir de 1920 había inaugurado el movimiento de las relaciones de objeto. En la idea de "La sombra del objeto cayó sobre el yo", del texto de Freud "Duelo y Melancolia", Melanie explora los mecanismos de identificación e introyección, y aunque de forma dudosa (Greenberg \& Mitchel, 1983) abre la puerta a la constitución del sujeto por la internalización del objeto. La cuestión 
radica en la duda de si la pulsión contiene en sí una preformación del objeto o si el objeto existe como realidad externa parcial y/o entera.

Simultáneamente Fairbairn profundiza esta idea y revisa la teoría psicoanalítica con la propuesta de que la pulsión busca el objeto y no el placer (Fairbain, 1944). El medio se valora como constituyente del sujeto.

Con Melanie Klein y Fairbairn, fundadores de la escuela de las relaciones de objeto, el self es determinado por los objetos internalizados y por la dinámica entre ellos, en el caso de Klein con un motor fantasmático y pulsional, en el caso de Fairbairn con una energía propia basada en búsqueda de relacionalidad.

En términos clínicos, se mantienen las reglas técnicas Freudianas. Se amplía el contenido de la interpretación, en el caso de Fairbairn, de forma radical, abriendo la puerta, aunque de forma dudosa, a la constitución del analista como buen objeto interno del paciente, pero en sustancia no se altera la centralidad del método clínico: la conciencia del inconsciente como forma de curación, ya sea libidinal u objetal, en una relación enmarcada en las reglas técnicas de Freud.

\section{II}

Es también en este momento que Winnicott comienza a trabajar con profundidad la idea de un analista función que repara una falla en el desarrollo del paciente. Winnicott (1964) nos dice que el bebé no existe, afirmación que tiene implícita una crítica. Si en Freud y Klein el bebé parece tener una unidad inicial, en Winnicott el bebé no existe sin el medio, el bebé se constituye en la relación con la madre. Winnicott desplaza el foco del mundo interno del bebé a la madre como constituyente del psiquismo y de la psicopatología. En este movimiento relativiza los principales conceptos del psicoanálisis, deja para segundo plano la sexualidad infantil, la madre como objeto pulsional y toda la mecánica pulsional de la metapsicología.

Para Winnicott (1988) la dependencia del recién nacido es absoluta, no sobrevive sin los cuidados del ambiente, una dependencia que el bebé desconoce pues considera como self todo lo que le rodea incluyendo a la madre. El desarrollo emocional sigue una línea de continuidad existencial que comprende las fases de dependencia total, dependencia relativa e independencia relativa, en estas fases ocurren tres procesos, el proceso de integración, el de personalización y el de realización. Es necesaria una madre suficientemente buena para que el movimiento de continuidad existencial se concretice, el bebé tiene el potencial, pero necesita el medio para activar ese potencial. 
La madre suficientemente buena, además de la preocupación materna primaria, una identificación con el bebé del orden del simbiótico que permite atender a sus necesidades, deberá ejercer tres funciones centrales: holding, handling y presentación del objeto. El holding es una función de sustentación, en la que la madre coloca al bebé en la vivencia de una rutina de cuidados y protección que lo sostienen psíquicamente. El handling está relacionado con el toque que armoniza la vida psíquica y el cuerpo.

Progresivamente el bebé va tomando conciencia del no yo, la constitución del yo es hecha por contraste con lo que es sentido como no-yo, depende en mucho de la capacidad de la madre para introducir elementos de separación, al no corresponder al deseo permite una salida de la omnipotencia. Entre el yo y el no-yo surge el espacio potencial, localización de objetos y fenómenos transitivos (espacio entre uno y otro donde se puede anclar la idea de tercero y de intersubjetividad). La existencia de este espacio significa la presencia de una madre suficientemente buena (Winnicott, 1971).

Las observaciones del desarrollo primitivo tienen impacto en el enfoque clínico, dice Winnicott (1984) que los pacientes que no tuvieron una madre suficientemente buena van a vivir en el análisis una relación de dependencia absoluta, regresiva, donde el analista queda en el lugar de la madre suficientemente buena. Se propone asegurar, en un nivel simbólico, la función de sustentación que posibilita desarrollar sentimientos de seguridad primitiva. Winnicott dice además que el analista se debe dejar usar por el paciente según sus necesidades terapéuticas. Se trata de una posición relacional muy diferente de la defendida por Freud. Como demuestran Black \& Mitchell (1995) Winnicott transforma la práctica psicoanalítica a partir de las características de la madre suficientemente buena.

Resaltemos una de las ideas con implicaciones clínicas, la idea de intrusión. Dice Winnicott que la madre suficiente buena incorpora el movimiento del bebé, se adapta a sus necesidades, se funda en este movimiento las bases del self verdadero, potenciador de gestos espontáneos y de sentimientos de autenticidad. Cuando la respuesta de la madre ignora al bebé hablamos de intrusión, movimientos que no respetan los tiempos y la espontaneidad del bebé, aquí el descubrimiento del niño es sustituido por el gesto de la madre y se interrumpe el proceso de continuidad existencial, el niño pasa a funcionar con movimientos de reacción y sumisión, las bases del falso-self. El niño sometido desconoce su deseo, el niño es la voluntad de la madre, la sumisión pasa a formar parte de su naturaleza, parte de sí no sabe de otra parte de sí espontánea y creativa. Renuncia a la esperanza de ver sus necesidades satisfechas y se adapta a lo que el medio le da.

Hay una posición relacional que valora el descubrimiento del bebé y otro que es una imposición y de donde deriva la idea de falso-self. Es aquí que la interpretación en el sentido 
Freudiano puede ser vista como una intrusión que lleva a la creación de un falso-self en el paciente, o sea, no se busca conocer el self del paciente, por el contrario se aplica una teoría al paciente a la cual él tiene forzosamente de adaptarse, siguiendo a Freud, se considera resistencia a la expresión del self espontáneo. La interpretación en Winnicott tiene que ser construida a dos, a la manera del squiggle game (Abello y Liberman, 2011).

Casement (2013) profundiza el concepto de intrusión y se refiere a los peligros de un dogma psicoanalítico al nivel de una religión, cosa que queda en el polo opuesto a lo mejor que el psicoanálisis nos puede ofrecer. Evidencia la necesidad de no sobrepasar la angustia de nuestra ignorancia con una postura de dueños de la verdad, que es una intrusión en el mundo interno del paciente de la cual resulta la creación de un falso-self y una idealización del psicoterapeuta, infiltrada en la fragilidad de lo paciente.

La idea de un psicoterapeuta que desempeña la función de madre suficientemente buena pretende retomar el desarrollo donde no fue exitoso por falla ambiental. Slochower (2018) refiere que la crítica relacional a este modelo se centró en la idea de que existe una infantilización del paciente colocado en una posición de dependencia desamparada que alimenta la superioridad del analista. Un paternalismo sin mutualidad intersubjetiva. Aparentemente Benjamín (2012) evidencia esta idea. La cuestión es la forma en que Winnicott atiende, o no, a la subjetividad de la madre y del analista más allá de la función. La idea estará en el paso de un modelo de compensar lo que el paciente no tuvo, para otro de transformar y expandir lo que el paciente tiene a través de una relacionalidad facilitadora del reconocimiento de la subjetividad de cada uno.

\section{III}

Por último, veamos una dimensión del psicoterapeuta como otro relacional provisto de subjetividad y pensemos lo que cambia en la ecuación terapéutica. La radicalidad clínica del psicoanálisis relacional está en desplazar el foco del análisis del mundo interno del paciente para el análisis de la relación terapéutica. No se trata de desvalorizar el mundo interno del paciente, se trata de estudiar la articulación del mundo interno del paciente con el mundo interno del psicoterapeuta en un reconocimiento mutuo (Benjamín, 2012) y en una relación muy específica, como es la relación psicoterapéutica, enmarcada en una determinada cultura social (Altman, 2011, Coderch, 2017).

Si aceptamos que en lo esencial la relación precoz es intersubjetiva, la madre hace al hijo que hace a la madre, en una co-creación interactiva de regulación mutua (Stern, 1985, Beebe \& Lachmann, 2002), entonces la relación terapéutica sólo puede ser comprendida 
estudiando la articulación de la subjetividad del psicoterapeuta con la del paciente. Por mucho que nos cueste entrar en la ecuación no somos coherentes si no lo hacemos. Es un desafío inmenso. Tal vez un ejercicio imposible, sin embargo, también es imposible estudiar la mente de una persona sin ser en interacción con la de otra persona (Mitchell, 1997).

La inclusión de la subjetividad del psicoterapeuta en la ecuación terapéutica obliga a una nueva conceptualización de algunos conceptos y abre puertas a otros que mejor expliquen la interacción terapéutica. Varias preguntas se plantean ante la crítica relacional: si el inconsciente no es pulsional en una dinámica de conflicto metapsicológico, entonces ¿de qué inconsciente hablamos? Si no es la interpretación clásica y el insight lo que transforman y si la contención terapéutica no es suficiente, entonces ¿qué transforma en la relación terapéutica?

Freud y los autores relacionales parten de puntos de vista diferentes. Freud conceptualiza lo humano en un conflicto interno que se articula con lo externo, el psicoanálisis relacional, no rechazando la tradición psicoanalítica, rechaza la idea de un inconsciente pulsional que existe dentro de nosotros de forma fija, estructurada y predeterminada.

Mitchell (1998) intenta una integración de varias perspectivas relacionales en la idea de matriz relacional. La matriz relacional puede ser una referencia para la comprensión de la experiencia humana y de los conceptos profundizados por el psicoanálisis relacional.

Mitchell utiliza la imagen de Escher Drawing Hands, en la que dos manos se dibujan simultáneamente, como metáfora de la relación entre la biología y las relaciones humanas en la constitución del sujeto. La estructura biológica actúa en el medio y es simultáneamente dibujada por el medio relacional, se trata de una dialéctica interactiva de transformaciones mutuas. Mitchell integra la pulsionalidad diciendo que el deseo se experimenta en el contexto de la relación y es ese contexto que lo define y significa. Así, si para Freud la mente surge de presiones endógenas, para el modelo relacional la mente surge de la interacción con otras mentes. Resalta Mitchell que la cuestión no es si Freud dio o no importancia al medio y a la cultura, que si dio, la cuestión está en la forma en que definió esa interacción para la constitución del sujeto.

Mitchell define la dinámica psíquica integrando tres áreas correspondientes a tres modelos psicoanalíticos: la Psicología del Self, las Teorías Interpersonales y las Teorías de las relaciones de objeto. Considera que para la comprensión del humano tendremos que atender a su matriz relacional que está compuesta por tres dimensiones indisociables: el sí 
mismo, el mundo objetal interno y el espacio de interacción con los demás. Estos tres elementos son piel, carne y hueso de un todo psíquico imposible de estudiar aisladamente.

En relación con las teorías de las relaciones de objeto internalizadas ya vimos las posiciones de Melanie Klein, Fairbairn y Winnicott. En resumen: las teorías interpersonales (a partir de los trabajos de Sullivan, Erich Fromm y Clara Thompson) ponen el foco del análisis en las relaciones interpersonales del paciente y en su contexto social y cultural. Por su parte, las teorías de la Psicología del Self (partiendo de los trabajos de Anna Freud, Hartmann, Spitz, Mahler y concretada por Kohut) buscan comprender la articulación y la adaptación del ego (como estructura metapsicológica) con el medio y con la pulsionalidad. Principalmente con Kohut $(1971,1977)$ se considera el Self como entidad independiente ( $y$ transversal) a todas las instancias de la estructura metapsicológica. Estudiando pacientes narcisistas Kohut identifica las necesidades del niño en la construcción de un Self cohesivo y suficientemente valorado. Es en este contexto que surge el método clínico de la empatía introspectiva como forma de, terapéuticamente, entender y atender las necesidades del Self del paciente. El foco está en el análisis del sí mismo y no en el conflicto intrapsíquico.

En la lectura integradora de Mitchell la relación con el otro, el objeto interno y el sí mismo se configuran de forma permanente en una dinámica abierta y no lineal de pasado, presente, interno y externo. Se entrelazan y se transforman, se regulan mutuamente de forma sutil y compleja. Es un modelo abierto que teniendo una estructura no es estructural en el sentido de una rigidez predefinida de repetición de patrones internalizados. El sentido del sí mismo es un proceso en transformación interconectado con el objeto interno y con las relaciones interpersonales, la dinámica de los objetos internos es evolutiva en la interacción entre las relaciones interpersonales y el sí mismo. En esta lectura lo intrapsíquico pasa de estructura arqueológica cerrada a un proceso abierto en permanente desarrollo.

El conflicto en la perspectiva relacional es inherente a la interacción de las diferentes dimensiones de relacionalidad. El sentido de sí busca expresarse de forma satisfactoria, pero encuentra conflictos de relacionalidad interna y externa, con los objetos internos y en las relaciones interpersonales, resulta de aquí el origen del sentir, del síntoma y de la psicopatología relacional. La matriz relacional tiene un sentido evolutivo inherente y una necesidad de estabilidad en la regulación mutua entre todos los elementos. Esta dinámica de proceso entre los elementos de la matriz se hace en una dialéctica entre dos dimensiones interconectadas: procesos explícitos y procesos implícitos (o inconscientes). Este es un inconsciente relacional y procesal diferente del inconsciente pulsional. 
En términos clínicos no estudiamos el individuo, estudiamos su matriz relacional en la relación con otras matrices relacionales, una matriz relacional no existe sola. El análisis del encuentro terapéutico se compleja porque estudiamos el encuentro de la matriz relacional del paciente con la del psicoterapeuta. Este encuentro es interactivo de influencia recíproca en una dialéctica entre procesos explícitos e implícitos.

La transferencia, en esta perspectiva, puede ser pensada con el concepto de conocimiento relacional implícito estudiado por el Boston Change Study Group. Lyons-Ruth (2010) se refiere a este concepto como un conjunto de estrategias relacionales aprendidas desde la primera infancia. Son modelos internos que resultan de la internalización de patrones relacionales tempranos, son generados por movimientos de regulación mutua imperecederos vivenciados en milésimas de segundo. Ocurren antes de la adquisición del lenguaje, son anteriores a la capacidad de evocar imágenes o representaciones verbales y no se les tiene acceso a ellos por la conciencia. Su forma de representación no está compuesta por imágenes o palabras sino por procedimientos que determinan el cómo estar con alguien. Son estos los modelos que sirven de base para establecer el contacto con la realidad, son un saber implícito con patrones más o menos rígidos actualizados permanentemente en la interacción relacional. Tal vez en este punto pudiera haber optado, de forma similar, por el concepto de principios organizadores y por la noción de inconsciente pre-reflexivo de Atwood y Stolorow (1992).

La transferencia, en este sentido, es la actualización de lo conocimiento relacional implícito en la relación con el psicoterapeuta. La cuestión que se plantea es que en el encuentro terapéutico no hay solo una transferencia, hay dos transferencias, la transferencia del paciente y la transferencia del psicoterapeuta, lo que tenemos en estudio es la articulación entre dos transferencias. No podemos aceptar una transferencia pura del paciente, en la medida en que la transferencia del paciente está inserta en la dinámica relacional con el psicoterapeuta. Por su parte, el término contratransferencia, como resonancia en el psicoterapeuta de la transferencia del paciente, no es suficiente para describir lo que sucede porque no contiene en su definición la subjetividad del psicoterapeuta (Mc Laughlin, 1981, Aron, 1996). Desde la perspectiva relacional el análisis transferencial y contratransferencial resulta incompleto porque supone una transferencia pura del paciente (desligada de la interacción con el psicoterapeuta) y no incluye la subjetividad del psicoterapeuta en el proceso relacional.

El concepto de enactment puede ayudar a describir el acontecimiento terapéutico. Bromberg (2011) relaciona este concepto con el trauma y la disociación. El trauma tiene el efecto de un tsunami, desestabiliza los aspectos identitarios con un afecto caótico superior 
al que la mente es capaz de procesar. La disociación es la forma de escape cuando no hay escapatoria posible, es la solución ante el terror de la disolución identitaria (recuerdo la teoría del trauma Ferenczi). La capacidad disociativa, en mayor o menor grado, tiene un carácter adaptativo. El enactment surge como un acontecimiento resultante del encuentro entre partes disociadas del psicoterapeuta y del paciente. Se crea una situación con significado inconsciente para terapeuta y paciente que se basa en estados disociados del self de los dos, puede estar anclado en el pasado pero siempre es algo nuevo. Renik (1993, 1996) con la idea de la irreductibilidad de la subjetividad del psicoterapeuta, ensancha el concepto y ve la interacción inconsciente como constante e inevitable, la psicoterapia es un gran e inevitable enactment y sólo el análisis de esa interacción permite su transformación (Ferenczi llegó a este punto y encontró como solución el análisis mutuo).

Sassenfeld (2010) señala que para la emergencia y resolución del enactment es necesario que el psicoterapeuta se olvide de los manuales de técnica clásica y tenga una actitud terapéutica basada en la autenticidad y la espontaneidad.

Subyacente al análisis de la interacción relacional inconsciente está el concepto de self disclosure. Este tema gana importancia a partir del momento en que sabemos que no es posible no estar en la relación terapéutica, la apertura de la subjetividad del psicoterapeuta no es una elección, es una inevitabilidad (es lo que dicen las teorías de la comunicación), lo que el psicoterapeuta dice y no dice, hace y no hace es revelador de lo que piensa y siente. Pensar que el paciente no interpreta (consciente e inconscientemente) el comportamiento del psicoterapeuta actuando en función de eso, es una infantilización del paciente poco aceptable. La cuestión es lo que haremos con la inevitabilidad del self disclosure y cuáles son las consecuencias relacionales de las actitudes terapéuticas que elegimos en cada situación clínica.

La interpretación como herramienta clave de la transformación es cuestionada por los estudios del Boston Change Study Group. Levenkron (2009) refiere que tradicionalmente el analista extrae patrones de significado que traduce verbalmente, a través de la interpretación, remitiendo al pasado y al mundo interno del paciente. Ésta es la profundidad psicoanalítica clásica. Sucede que el saber relacional implícito, por sus características precoces y experienciales, no puede, en esencia, ser pensado ni verbalizado. Lo esencial de las transformaciones en los procedimientos relacionales internos, en la memoria implícita, no resulta del pensamiento consciente sino de la experiencia, lo que limita la idea de la interpretación como factor clave de la transformación.

El análisis conjunto del enactment es a través de la respuesta conjunta a la pregunta: ¿qué pasa aquí entre nosotros?. Por su carácter experiencial, permite acceder más fácilmente a 
los procesos relacionales implícitos y tiene un mayor potencial transformador de la relacionalidad interna y externa del paciente. Es en este sentido que se refiere a la confusión entre lo superficial y profundo, el potencial transformador está más en la apariencia de superficialidad que en la elocuencia interpretativa de lo profundo.

Podemos pensar, desde la lectura mediante la matriz relacional de Mitchell, que la interpretación colocada bajo la forma de iluminación del inconsciente, actúa sobre el sentido del sí mismo. La autoridad de la verdad, además de no transformar el conocimiento relacional implícito, coloca el sí mismo en una posición relacional de adaptación a la inferioridad y sumisión, anulando su capacidad de agencia. En última instancia se puede repetir el patrón relacional que se pretende transformar por la interpretación.

Es en este contexto que ganan sentido los conceptos de tercero analítico en la perspectiva de Ogden (2004) o de Benjamin (2012). Un espacio tercero intermedio y virtual construido por paciente y analista. Es en este espacio, en gran parte inconsciente, que están articuladas a subjetividades de cada uno. La comprensión del paciente viene de la comprensión de la articulación relacional con el psicoterapeuta. Como dice Ogden (2004), pensando en Winnicott, no existe paciente sin analista ni analista sin paciente.

Pienso que en todas las estructuras que vamos a construir para intentar comprender la relación terapéutica hay un elemento móvil y difícil de definir pero que alimenta todos los elementos de esas estructuras: la emoción. La emoción está en la relación terapéutica como la sangre está en nuestro cuerpo. Cuando intentamos percibir cuáles son los factores de la relación humana con potencial terapéutico muchos autores (Ávila Espada, 2013, Lachman, 2007, Coderch y Espinosa, 2016) anclados en estudios de la vinculación y en la experiencia clínica señalan que más importante que el insight racional es la conexión y la regulación emocional con el paciente.

Lewis Aron (cf. Naranjo, 2010) cuenta que Freud, quien fue analista de Ferenczi, durante ese análisis Ferenczi le dijo a Freud que se quería sentir emocionalmente ligado a él, y Freud interpretó en términos libidinales como una tendencia homosexual, "crece y se un hombre", dijo Freud. Ferenczi buscaba una experiencia emocional, Freud respondió con una interpretación racional y sexual. Este episodio simboliza las controversias entre los dos. En la nota de 19 de junio del Diario Clínico Ferenczi habla de la necesidad de un amor analítico que sirve de contrapeso a las situaciones traumáticas, dice que ningún análisis podrá realmente tener éxito si no llegamos a amar al paciente, en la nota de 11 de agosto dice que el "el objetivo final de un análisis didáctico es que el futuro analista sea capaz de salir de su narcisismo y amar a los demás". 
La afectividad y los procesos emocionales están entrelazados en todas las dimensiones relacionales, y es en la dimensión emocional que se anclan los procesos de vinculación, la cognición, la memoria, la formación del self (Coderch y Espinosa, 2016). Las emociones son indisociables de la experiencia humana y son el motor de la relacionalidad. Véase el libro de António Damasio con el sugestivo título El error de Descartes (1994) donde nos quedamos con la idea de que el pensamiento baila la música de las emociones.

Lachman (2007) respondiendo a la pregunta "¿cómo ocurren las transformaciones?" enumera tres principios fundamentales: regulaciones mutuas continuas; interrupción y reparación de las regulaciones mutuas; y momentos afectivos profundos. La psicoterapia es un progreso relacional, una intimidad de regulación mutua, en última instancia, afectiva. El motor de esta regulación mutua es la capacidad de expresión afectiva y la conexión emocional empática. Las reglas clásicas cierran esta puerta, pero aun sin fundamentación teórica, fácilmente caímos en un racionalismo teórico defensivo de la intimidad, del sufrimiento del paciente y de los sentimientos que surgen entre nosotros y los pacientes. En el polo opuesto tendríamos la intimidad intrusiva que no respeta tiempos y límites del paciente (recordemos el tacto psicológico de Ferenczi).

La regulación emocional pasa muchas veces por expresiones corporales que contienen mensajes emocionales implícitos, acercamientos y alejamientos, pequeñas sutilezas, un tono de voz, una mirada, un toque, un saludo diferente. Todos los sentimientos pasan por la relación terapéutica a través de la verbalización, pero principalmente por movimientos imperceptibles de auto y de mutua regulación que definen un ambiente afectivo. Tal vez el análisis de la regulación emocional mutua pueda tener potencial de transformación en la relacionalidad interna y externa del paciente. Buechler $(2012,2015)$ habla del papel crucial de la implicación emocional del analista con el paciente y considera que la vivencia y análisis de sentimientos como la alegría, la vergüenza, el odio o el miedo en el paciente, el analista y la dinámica entre los dos es fundamental.

En este ámbito gana significado la idea de mutualidad (Aron, 1996) que reconoce la necesidad de colaboración mutua, en un trabajo hecho a dos, co-creado, esta mutualidad, porque está integrada en una relación asimétrica por naturaleza, contiene en sí un valor terapéutico. Aron recuerda a Ferenczi cuando decía que la humildad del analista y el reconocimiento de sus dificultades tenía un efecto terapéutico en los pacientes.

Entrelazado en este tema está el de la autoridad del psicoterapeuta, la cuestión no es tanto una autoridad dueña de la verdad sino una autoridad derivada del reconocimiento de la capacidad de mantener un registro relacional transformador. Tenemos la autoridad de la responsabilidad, sabemos de las consecuencias de las dinámicas relacionales de 
dominación y sumisión, y por eso la mutualidad clínica es relevante. O no fueran nuestros pacientes humanos, exactamente tanto como nosotros.

En este encuentro de matrices relacionales, la cuestión de la técnica, como método predeterminado, pierde algún sentido porque navegamos en lo desconocido, cada matriz relacional es única, el campo donde trabajamos es único, nunca vino en ningún libro. Tenemos nuestras brújulas, pero la irreductibilidad de la subjetividad del paciente y del psicoterapeuta pone en cuestión la idea de técnica. Orange, Atwood y Stolorow (1997) dicen que este trabajo es más una práctica en la medida en que el encuentro de dos subjetividades deja en abierto un campo indefinido e imprevisible que no es consecuencia de una técnica a priori. La técnica a priori distorsiona el campo y lo cierra.

Pensando en Klein y Freud diríamos que, rompiendo con el positivismo científico de la época, estos autores constituyeron una subjetividad (que daba sentido a los síntomas) para los pacientes, sin embargo, luego encerraron los pacientes dentro de la subjetividad que para ellos construyeron.

La perspectiva relacional nos obliga a reflexionar sobre reglas y hábitos que tenemos como ciertos, no existen soluciones universales y cada relación, en cada momento, exige de nosotros negociación. La negociación sincera, justa y honesta puede ser en sí misma terapéutica. Con cada paciente tendremos que crear un nuevo marco, un nuevo proceso, nuevos límites y nuevas reglas organizadoras de la experiencia terapéutica. En este sentido Bacal (2017) habla de una responsividad óptima del psicoterapeuta, derivada de la relación con cada paciente y de cada situación clínica, y de una teoría de la especificidad que remite para el análisis de la singularidad de cada relación terapéutica.

El psicoterapeuta nace cuando deja de ser una pantalla proyectiva, una función o una caja de resonancia del mundo interno del paciente para convertirse en un constituyente del espacio terapéutico. Todos pensamos en la mejor forma de ayudar a nuestros pacientes. La respuesta, nuestro entendimiento, viene de la complejidad de nuestra subjetividad, Atwood y Stolorow en Faces in a Cloud (1979) demuestran que las conclusiones teóricas a las que llegaron autores como Freud o Jung están relacionadas con su propia subjetividad, es decir que las conclusiones a las que llegamos sobre el paciente y la relación terapéutica resultan de nuestra subjetividad. No creo que esto sea un problema, puede serlo, si no somos conscientes de esta inevitabilidad.

Hay que tener en cuenta nuestras fragilidades, nuestros traumas, nuestro narcisismo y nuestros estados fascistas de la mente (Bollas, 1994), lo peor que nos puede pasar es pensar que eso sólo afecta a los demás, que estamos resueltos o curados. Necesitamos superar el mito de que en la psicoterapia hay una persona enferma y otra sana, la complejidad de lo 
humano no permite esa dicotomía y en honor a la verdad no sabemos lo que es una cura en psicoanálisis, sólo tenemos como objetivo proporcionar a la persona que nos busca niveles de relacionalidad interna y externa más satisfactorios.

Creo que es de la conciencia de nuestras dificultades que puede derivar nuestro potencial: una ética relacional de la que resulte una relación humana lo más justa posible, que contenga en sí misma potencial terapéutico, quien sabe si el único potencial terapéutico que tenemos, no supimos nosotros de la importancia de la relación en el sufrimiento y en la psicopatología. Una relacionalidad democrática, en una intimidad terapéutica entre diferentes matrices relacionales en que una está atenta a las dinámicas implícitas e implicada emocionalmente con el sufrimiento de la otra.

No hay relación de ayuda ni teoría segura si no tiene por principio la humanidad y la autenticidad posible. El registro de uno sobre otro, un yo que sabe y otro que no sabe, mantiene o implementa un registro de adaptación y falso-self (Casement, 2013) y de dominio y sumisión (Benjamín, 1988).

Orange $(2011,2016)$ habla de la necesidad de un cambio ético en psicoanálisis, basado en la hospitalidad, solidaridad y responsabilidad ante el otro que sufre, una hermenéutica de la confianza por oposición a la hermenéutica de la sospecha, la sospecha de la pulsionalidad del paciente, la sospecha de la psicopatología. Dice que la resistencia del paciente a la verdad del analista no es una defensa de motivaciones inconscientes inferiores, ni la protección de un ego frágil, sino una lucha por la supervivencia psicológica.

La responsabilidad ética de Orange ante un hermano que sufre y que debe ser recibido con hospitalidad en un reconocimiento y valorización de su subjetividad encuentra paralelismos en la filosofía ancestral Africana Ubuntu seguida, por ejemplo, por Nélson Mandela. Ubunto no es una teoría filosófica (como sucede en las teorías filosóficas de las sociedades occidentales). Ubunto es una práctica filosófica que viene de la relación entre las personas, y que, curiosamente, tiene subyacente la idea de intersubjetividad (yo soy porque tú eres, yo sólo puedo ser a través de las otras personas). Esta conciencia intersubjetiva apela a la responsabilidad sobre el otro, nosotros somos los otros. Es muy interesante que la ancestralidad africana tuviera en sus principios relacionales comunitarios la idea de intersubjetividad y responsabilidad ante el otro, África, luego víctima de la intrusión destructiva del colonialismo Europeo que se creía superior y dueño de la verdad.

Si Freud se colocaba ante la psiquiatría en una posición de recuperación de la subjetividad que la ciencia positivista anulaba en los pacientes (en la búsqueda de una localización cerebral para los síntomas), al mismo tiempo que se colocaba ante la sociedad en un desafío a la época victoriana defendiendo la expresión de la sexualidad, tal vez hoy el 
psicoanálisis pueda colocarse ante la sociedad en una posición de responsabilidad ética ante el otro, en la idea de intersubjetividad en la que todos somos responsables unos por otros.

Tal vez el psicoanálisis deba apelar a una sociedad donde nadie puede estar contento con la humillación del otro. Esto porque sabemos (por la evolución de la historia del psicoanálisis y de sus conocimientos teóricos) que es la humillación, la negación y la desvalorización de la subjetividad del otro que provoca ciclos de sufrimiento psíquico.

Más importante que la competición es la cooperación. Vivimos de principios totalizadores (desde los vencedores y los perdedores a las etiquetas psicopatológicas) que anulan la subjetividad del otro, objetivan y en último análisis pueden destruir en un mecanismo que Bollas (1994) describió como Estados Fascistas de la Mente. Dentro y fuera de la relación terapéutica tenemos la responsabilidad de lo que sabemos en lo que hacemos.

Un último punto de reflexión clínica, Mitchel (1988) señala que el abandono de la técnica clásica generó el miedo al vale todo, como si al abandono de la autoridad clásica, de la neutralidad, del anonimato y abstinencia sólo pudiera corresponder la irresponsabilidad. Es exactamente la responsabilidad la que lleva a cambiar de actitud terapéutica. Sería irresponsable si no tuviera la flexibilidad de convertirme en lo que pienso que es mejor para los pacientes, aunque lo haga con asombro y miedo.

Por otro lado, Slochower (2018) dice que toda la teoría psicoanalítica tiene un ideal clínico construido en guerra con ideales clínicos de otras teorías, polarizamos, elevamos nuestra posición, estereotipamos y denegamos a los demás. El psicoanálisis no pensaba la subjetividad del analista, fue precisamente ahí donde el paradigma relacional fue hacer su ideal, donde antes estaba la necesidad de más análisis ahora está el oro puro de la clínica. En una reciente edición con el interesante título De-Idealizing Relational Theory. A Critique From Within, Corbett (2018) llama la atención sobre la excesiva valorización de la espontaneidad del psicoterapeuta que olvida la necesidad de privacidad y de un espacio de contemplación. Slochower (2018) valora la necesidad de colocar entre paréntesis la subjetividad para ejercer funciones de holding. Añade que la mutualidad clínica no es un dato adquirido, sino un camino.

Concluyendo, el psicoanálisis está en constante transformación, más hoy que ayer, pero sin duda desde el principio, y es en la relación humana, en sus múltiples dimensiones, que la teoría y la práctica psicoanalítica ponen la clave en el entendimiento de lo que somos, de lo que fuimos y de lo que podremos ser. Es en la relación humana que crecemos y nos transformamos y por eso sólo con el estudio de la relación humana, y la relación 
psicoterapéutica es evidentemente una relación humana, podremos tener esperanza en contribuir a la transformación de aquellos que, por su sufrimiento, nos buscan.

\section{REFERENCIAS}

Abello Blanco, A. \& Liberman, A. (2011). Una Introduccion a la obra de D. W. Winnicott. Contribuiciones al pensamento relacional. Madrid: Ágora Relacional

Altman, N. (2011). El psicoanálisis en el contexto cultural. Clínica e Investigación Relacional, 5 (3): 389-402. [Recuperado de www.ceir.org.es ]

Aron, L. \& Harris, A. (1993). Discover and Rediscover. In The Legacy of Sandor Ferenczi, Ed. Aron \& Harris. Hillsdale: The Analitic Press.

Aron, L. (1996). Meeting of minds. Mutuality in psychoanalysis. Hillsdale: The Analytic Press.

Atwood G. \& Stolorow R. (1992). Los contextos del ser. Las bases intersubjetivas de la vida psíquica. Barcelona: Herder Editorial. (2004).

Atwood, G. Orange, \& D. Stolorow, R. 1997). Trabajando intersubjetivamente. Contextualismo en la parática psicoanalítica. Madrid: Ágora Relacional. (2012)

Atwood, G., Orange, \& D. Stolorow, R. (1997). Trabajando intersubjetivamente. Contextualismo en la parática psicoanalítica. Madrid: Ágora Relacional. (2012)

Ávila Espada, A. (2013). La relación, contexto determinante de la transformación.Temas de Psicoanálisis. N. 6. Julho de 2013.

Ávila Espada, A. (2013). Las origenes de la prespectiva interpersonal e sócio-cultural. En La Tradicion interpersonal, Perspectiva social e cultural en psicoanálise. Alejandro Avila Espada (Ed). Madrid: Ágora Relacional.

Bacal, H.A. (2017). La especificidad de la eficacia terapéutica en el tratamiento psicoanalítico. Clínica e Investigación Relacional, 11 (2): 217-231. [Recuperado de www.ceir.info]

Beebe, B. \& Lachmann, F. (2002). Infant Research and Adult Treatment: Co-constructing Interactions. London: Analitic Press.

Benjamin, J. (1988). Los Lazos del Amor. Psicoanalisis, feminismo e el problema de la dominacion. Buenos Aires: Paidos.

Benjamin, J. (2012). El Tercero. Reconocimiento. Clínica e Investigación Relacional, 6 (2): 169-179. [Recuperado de www.ceir.org.es ]

Black, M. e Mitchell S. (1995). Más allá de Freud. Una Historia del pensamiento psicoanalitico moderno. Barcelona: Herder Editorial. 2004.

Bollas, C. (1994). Ser un personaje: psicoanálisis y experiencia del sí-mismo. Buenos Aires: Paidos.

Bromberg, P. (2011). La sombra del tsunami y el desarrollo de la mente relacional. Madrid: Ágora Relacional. (2017). 
Buechler, S. (2012). Déficits de autoestima en una sociedad cambiante. Clínica e Investigación Relacional, 6 (1): 52-XX. [Recuperado de www.ceir.org.es ]

Buechler, S. (2015). La alegría, la vergüenza, el arrepentimiento y la tristeza en la terapia. Clínica e Investigación Relacional, 9 (2): 345-357. [ISSN 1988-2939] [Recuperado de www.ceir.org.es]

Casement (2013). Further Learning from the Patient: The analytic space and process. London: Routledge.

Corbett, K. (2018). The analyst's private space: spontaneity, ritual, psychotherapeutic action, and sel-care. In De-Idealizing Relational Theory. A Critique From Winthin. Ed. Aron, Grand \& Slochower. New York: Routledge.

Coderch, J. \& Espinosa, A. (2016) Emoción e relaciones humanas. El psicoanálisis relacional como terapêutica social. Madrid: Ágora Relacional.

Coderch, J. (2012). La relacion paciente-terapeuta. El campo del psicoanalisis e la psicoterapia psicoanalitica. Barcelona: Herder Editoral.

Coderch, J. (2014). Avances en psicoanalisis relacional. Nuevos campos de explóracion para el psicoanálisis. Madrid: Ágora Relacional.

Coderch, J. (2010). La Prática de la psicoterapia relacional. El modelo interactivo en el campo del psicoanálisis. Madrid: Ágora Relacional.

Coderch, J. (2017). Comprendiendo a una sociedad en cambio para comprender a los pacientes. Clínica e Investigación Relacional, 11 (1): 51-69. [Recuperado de www.ceir.org.es ]

Damásio, A. (1994). O Erro de Descartes. Emoção, Razão e Cérebro Humano.Lisboa: Publicações Europa-América.

Etchegoyen, H. (1986). Los Fundamentos de la técnica psicoanalitica. Buenos Aires: Amorrortu Editores.

Fairbain, R. (1944). Estrutura endopsiquica considerada em termos de relações de objecto. In Estudos Psicanalíticos da Personalidade. Lisboa: Vega Editora.

Ferenczi, S. (1929) El niño mal recibido y su impulso de muerte. Psicoanálisis IV. Madrid: EspasaCalpe. (1984). pag. 85-90.

Ferenczi, S. (1933). Confusion de lengua entre los adultos y el niño. Psicoanálisis IV. Madrid: EspasaCalpe. (1984). pag. 139-149.

Ferenczi, S. (1988). The Clinical Diary of Sándor Ferenczi. Cambridge, Massachusetts and London: Harvard University Press.

Freud, S. (1887-1904). A Correspondência Completa de Sigmund Freud para Wilhelm Fliess. Edição de Jeffrey Masson. Rio de Janeiro: Imago Editores (1986).

Freud, S. (1893). Sobre o mecanismo psíquico dos fenômenos histéricos: uma conferência. Edição Standard brasileira. Rio de Janeiro: Imago. (1987) V. III. p. 33-47. 
Freud, S. (1894). As Neuropsicoses de Defesa. Edição standard brasileira. V. III. Rio de Janeiro: Imago. (1987). p. 49-65.

Freud, S. (1895a). Estudos sobre a Histeria. Edição standard brasileira. Vol.VII. Rio de Janeiro: Imago (1987).

Freud, S. (1896a). A hereditariedade e a etiologia das neuroses. Edição Standard brasileira. Rio de Janeiro: Imago. (1987) V. III, p.133-148.

Freud, S. (1900). A Interpretação dos Sonhos. Edição standard brasileira. Vol. IV e V.Rio de Janeiro: Imago (1987).

Freud, S. (1904). O método psicanalítico de Freud. Edição standard brasileira. Vol.VII. Rio de Janeiro: Imago (1987), p.231-238.

Freud, S. (1905). Três ensaios sobre a sexualidade. Edição standard brasileira. Vol.VII. Rio de Janeiro: Imago (1987) p.117-230.

Freud, S. (1912a). A dinâmica da transferência. Edição Standard brasileira. Rio de Janeiro: Imago, 1987. Vol. XII, p.129-143.

Freud, S. (1912b). Recomendações aos médicos que exercem a psicanálise. Edição Standard brasileira. Rio de Janeiro: Imago, 1987. Vol. XII, p.145-159.

Freud, S. (1913). Sobre o início do tratamento. Edição Standard brasileira. Rio de Janeiro: Imago, 1987. Vol. XII, p. 161-187.

Freud, S. (1914a). Recordar, repetir e elaborar. Edição standard brasileira. Vol. XII. Rio de Janeiro: Imago, 1990, p. 189-203.

Freud, S. (1925). A Negativa. Edição standard brasileira. Vol. XIX. Rio de Janeiro: Imago (1996). p. 261-269.

Greenberg, J., \& Mitchell, S. (1983). Relações de Objecto na Teoria Psicanalítica. Lisboa: Climepsi Editores. 2003..

Heimann, P. (1950). On Counter-Transference. International Journal of Psycho-Analysis, Vol. 31. p.81-84.

Kohut, H. (1971). Análisis del Self. El tratamento psicoanalitico de los transtornos narcisistas de la personalidade. Buenos Aires: Amorrotu Editores. (1977)

Kohut, H. (1977). A Restauração do Self. Rio de Janeiro: Imago Editora. (1988)

Lachmann, F. (2007). El proceso de transformar. Clínica e Investigación Relacional, 1 (1): 42-52. [Recuperado de www.ceir.org.es ]

Levenkron, H. (2009). Abordando lo implícito. Puntos de encuentro entre el Grupo de Boston para el estudio del proceso de cambio y el psicoanálisis relacional. Clinica e investigación relacional, 3 (3): 495-524 [Recuperado de www.ceir.org.es ]

Little, M. (1951). Counter-Transference and the Patient's Response to It. Int. J. Psycho-Anal., 32:32-40. 
Lyons-Ruth, K. (2010). El desarrollo de los conflictos y las defensas en los procesos relacionales implícitos. Clínica e Investigación Relacional, 4 (2): 321-335. [Recuperado de www.ceir.org.es ]

Martínez Ibáñez, J. J. (2017). La Mente y el inconsciente relacional. Clínica e Investigación Relacional, 11 (2): 352-368. [Recuperado de www.ceir.org.es ]

Mc Laughlin, (1981). Transference, Psychic Reality, and Countertransference. The Psychoanalytic Quarterly, 50(4):639-64.

Mitchell, S. (1988). Relational Concepts in Psychoanalysis: An Integration. Cambridge: Harvard University.

Mitchell, S. (1997). Influence and autonomy in psychoanalysis. Hillsdale: The Analitic Press.

Naranjo, R. (2010). Entrevista a Lewis Aron. Clínica e Investigación Relacional, 4 (3): 678-684. [Recuperado de www.ceir.org.es ]

Ogden, T. (2004). The Analytic Third: Implications for Psychoanalytic Theory and Technique, The Psychoanalytic Quarterly, 73:1, 167-195,

Ogden, T. (2010). Esta arte da psicanálise - Sonhando Sonhos Não Sonhados e Gritos Interrompidos. Porto Alegre: Artmed.

Orange, D. (2011). "La actitud de los héroes": Bernard Brandchaft y la hermenéutica de la confianza. Clínica e Investigación Relacional, 5 (3): 507-515. [Recuperado de www.ceir.org.es ]

Orange, D. (2016). El guardián de mi hermano. Recursos para un giro ético en Psicoanálisis. Clínica e Investigación Relacional, 10 (1): 11-2. [Recuperado de www.ceir.org.es ]

Orange, D. (2016). Una actualización: de la Teoría de los Sistemas Intersubjetivos al Giro Ético en Psicoanálisis, CelR, pp. 27-48.[Recuperado de www.ceir.org.es ]

Racker, H. (1941). "Neurose de Contratransferência". In Estudos sobre Técnica Psicanalítica. Porto Alegre: Artes Médicas. (1982)

Renik, O. (1993). Analytic interaction: Conceptualizing technique in light of the analyst's irreducible subjectivity. The Psychoanalytic Quarterly, 62 (4), 553-571.

Renik, O. (1996). The perils of neutrality. The Psychoanalytic Quarterly, vol. LXV, N. 3, págs. 495517 (1996)

Sassenfeld J., A. (2010). Enactments: Una perspectiva relacional sobre vínculo, acción e inconsciente. Clínica e Investigación Relacional, 4 (1): 142-181. [Recuperado de www.ceir.org.es]

Slochower, J. (2018). Going too far: relational heroines and relational excess. In De-Idealizing Relational Theory. A Critique From Within. Ed. Aron, Grand \& Slochower. New York: Routledge.

Stern, D. N. (1985). El mundo interpersonal del infante: una prespectiva desde la psicoanalisis y la psicologia evolutica. Buenos Aires: Paidos. (1991)

Stolorow, R. \& Atwood, G. (1979). Faces in a cloud: Subjectivity in personality theory. Northvale: Aronson. 
Stolorow, R. \& Atwood, G. (1992). Los Contextos del Ser. Las bases intersubjetivas de la vida psíquica. Barcelona: Herder Editorial. (2004)

Stolorow, R. \& Atwood, G. (2013). Deconstruyendo el mito del analista neutral: Una alternativa desde la Teoría de los Sistemas Intersubjetivos. Clínica e Investigación Relacional, 7 (1): 60-74. (original de 1997). [Recuperado de www.ceir.org.es ]

Stolorow, R.D., Orange, D.M. y Atwood, G.E. (2012). Horizontes del Mundo. Una alternativa post-Cartesiana al Inconsciente Freudiano. Clínica e Investigación Relacional, 6 (3): 434451. [Recuperado de www.ceir.org.es]

Winnicott, D. (1971). O Brincar e a Realidade. Rio de Janeiro: Imago Editora. (1975).

Winnicott, D. (1947). O Ódio na contratransferência. In Da Pediatria à Psicanálise. Obras Escolhidas. Rio de Janeiro: Imago Editora. (2000).

Winnicott, D. (1964). The Child, the Family and the Outside World. Londres: Pelican Books.

Winnicott, D. (1984). Deprivación e Delincuencia. Buenos Aires: Paidos. 1990.

Winnicott, D. (1988). Da Pediatria à Psicanálise. Obras Escolhidas. Rio de Janeiro: Imago Editora. (2000).

Winnicott, D. (1988). Natureza Humana. Rio de Janeiro: Imago Editora.

Original recibido con fecha: 26/9/2018 Revisado: 15/03/2019 Aceptado: 30/03/2019

En este mismo número de CeIR se publica online la versión original portuguesa de este trabajo. 